Tạp chí Khoa học và Công nghệ biển T10 (2010). Số 3. Tr 15 - 32

\title{
XÂY DỰNG ĐIỀU KIỆN BỀN MỞ RộNG ĐỂ XÁC ĐỊNH Độ TIN CẬY TỔNG THỂ ĐÁNH GIÁ AN TOÀN CỦA KẾT CẤU CÔNG TRÌNH BIỄN CỐ ĐỊNH BÀNG THÉP, ÁP DỤNG CHO ĐIỀU KIỆN BIỂN NƯỚC SÂU VIỆT NAM
}

\author{
PHẠM KHÁ̉C HÙNG
}

Viện Xây dụng Công trình biển, Trường Đại học Xây dựng

\begin{abstract}
Tóm tắt: Bài báo trình bày một cách đánh giá mới về an toàn của kết cấu công trình biển cố định bằng thép kiểu Jacket phục vu khai thác dầu khí ở vùng nước sâu, dựa trên độ tin cậy tổng thể của kết cấu được xác định theo "điều kiện bền mở rộng", là điều kiện bền có kể đến hiện trạng kết cấu bị phá huỷ do mỏi tích luỹ trong quá trình khai thác.

Với điều kiện bền mở rộng, việc đánh giá an toàn của kết cấu chịu tác động của tải trọng sóng ngẫu nhiên theo độ tin cậy tổng thể cho kết quả chính xác hơn so với cách đánh giá theo điều kiện bền độ bền truyền thống trong các Tiêu chuẩn thiết kế hiện hành, đặc biệt hưu hiệu đối với các công trình biển nuớc sâu.

Kết quả nghiên cứu đã được bước đầu áp dụng vào điều kiện thưc tế của vùng biển sâu tới $200 \mathrm{~m}$ thuộc bể Nam Côn Son, thềm luc địa Đông-Nam Việt Nam. Bài báo này được trích tì̀ kết quả nghiên cứu của đề tài cấp Nhà nuớc KC.09.15/06-10, do tác giả làm chủ nhiệm đề tài trục tiếp thực hiện với sự cộng tác của một số đồng nghiệp tại Viện Xây dưng Công trình biển.
\end{abstract}

\section{MỞ ĐẦU}

Trong tính toán thiết kế kết cấu công trình biển (CTB) cố định kiểu Jacket (là kiểu kết cấu chân đế được sử dụng phổ biến trong $\mathrm{CTB}$ cố định bằng thép), kiểm tra bền là bài toán được thực hiện trước tiên bên cạnh bài toán kiểm tra mỏi [2] để đánh giá an toàn của các kết cấu CTB. Việc kiểm tra kết cấu CTB theo điều kiện bền được thực hiện theo “Trạng thái giới hạn cực đại” (Ultimate Limit State - ULS), nhằm đảm bảo kết cấu khai thác được an toàn trong điều kiện môi trường cực trị (sóng bão 100 năm hoặc 50 năm, tuỳ theo tiêu chuẩn thiết kế).

Điều kiện bền cũng là cơ sở để xác định cấu hình kết cấu khối chân đế (KCĐ) Jacket của $\mathrm{CTB}$ cố định bằng thép, sau quá trình lặp và điều chỉnh để có kích thước kết cấu hợp lý, thoả mãn "2 mục tiêu: an toàn và tiết kiệm vật liệu ".

Mục tiêu tiết kiệm vật liệu được xác định bởi "Hệ số sử dụng thép"- SUF (Steel Utilization Factor): 


$$
\mathrm{SUF}=\frac{A}{B} \quad==>\operatorname{Min}(*)
$$

trong đó: + A - Tổng trọng lượng thép của kết cấu chịu lực (chủ yếu là $\mathrm{KCĐ);}$

+ B - Tải trọng hữu ích của khối thượng tầng đặt lên kết cấu chịu lực KCĐ.

Một số thiết kế điển hình cho loại giàn đa chức năng (Công nghệ-Khoan-Người ở:PDQ) đã đạt SUF = 40 - $45 \%$ [12].

Tuy nhiên việc chọn cấu hình hợp lý, ngoài chỉ tiêu (*), cũng cần xét đến các yếu tố khác để đảm bảo giá thành công trình chấp nhận được, như chi phí thi công, giá thành nguyên vật liệu, chi phí trong quá trình duy tu bảo dưỡng công trình.

Ngày nay do nhu cầu năng lượng tăng mạnh, xu thế chung của thế giới là khai thác dầu khí biển ngày càng ra xa bờ, với độ sâu nước ngày càng tăng. Hiện nay, độ sâu nước khai thác đã đạt $2400 \mathrm{~m}$, trong đó CTB cố định kiểu Jacket đã đạt tới $412 \mathrm{~m}$ nước, xem hình 1 [10].

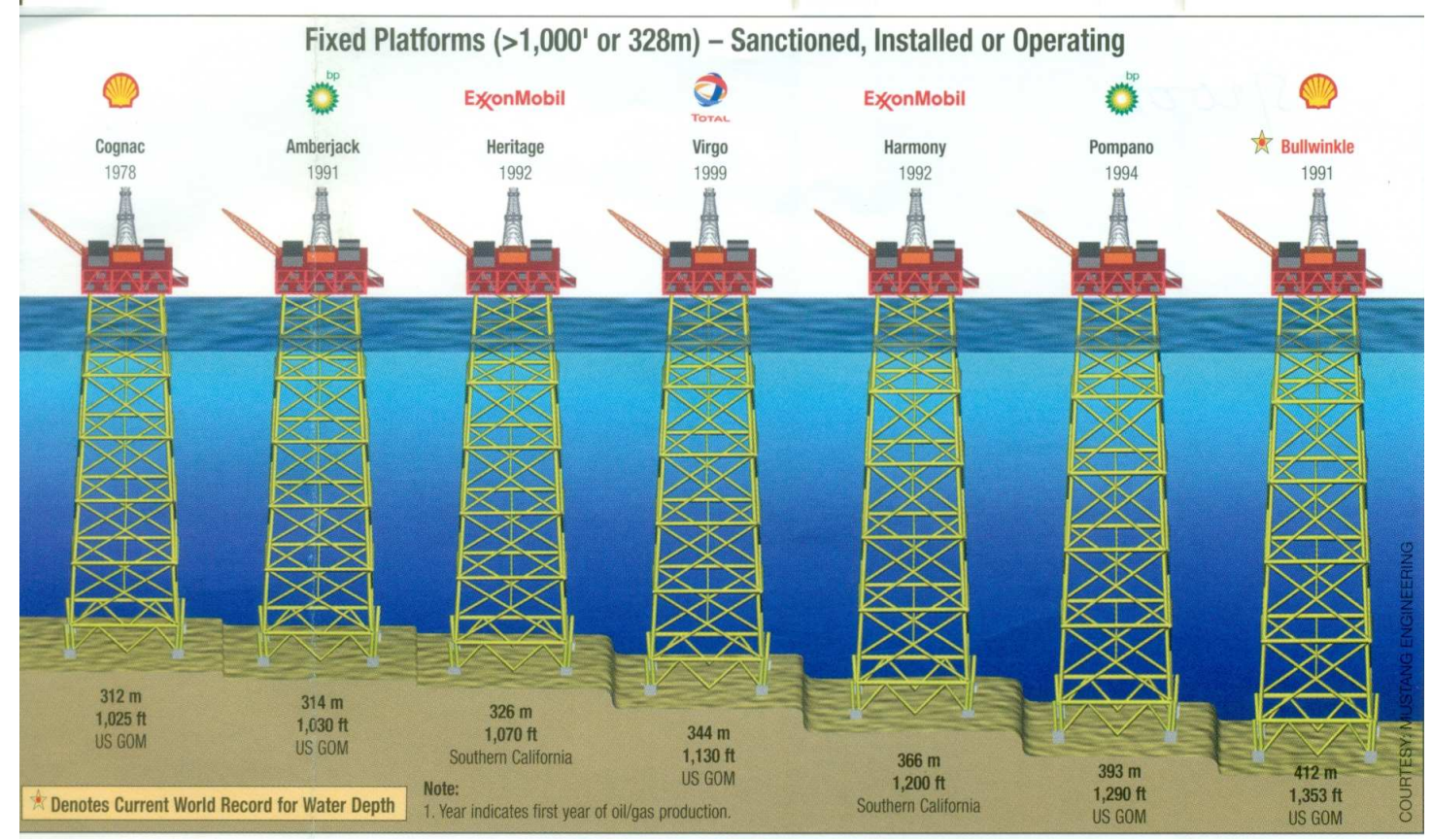

Hình 1: Sơ đồ các kết cấu Jacket của CTB cố định đã được xây dựng ở vùng nước sâu (lớn hơn $1000 \mathrm{ft}$ ) trên Thế giới

Ở nước ta, mục tiêu chiến lược của ngành dầu khí Việt Nam là "Đẩy mạnh tìm kiếm thăm dò, gia tăng trữ lượng có thể khai thác, ưu tiên phát triển những vùng biển nước sâu, xa bờ; Phấn đấu khai thác 25 - 35 triệu tấn quy dầu/năm" [6]. 
Trên hình 2 đưa ra kết quả nghiên cứu đánh giá tiềm năng dầu khí vùng nước sâu TLĐ.VN, điển hình là Bể Phú Khánh (khu vực 1) và bể Tư Chính - Vũng Mây \& Tây Nam quần đảo Trường $\mathrm{Sa}$ (khu vực 2), với độ sâu nước từ $200 \mathrm{~m}$ đến trên 1000 m [7].

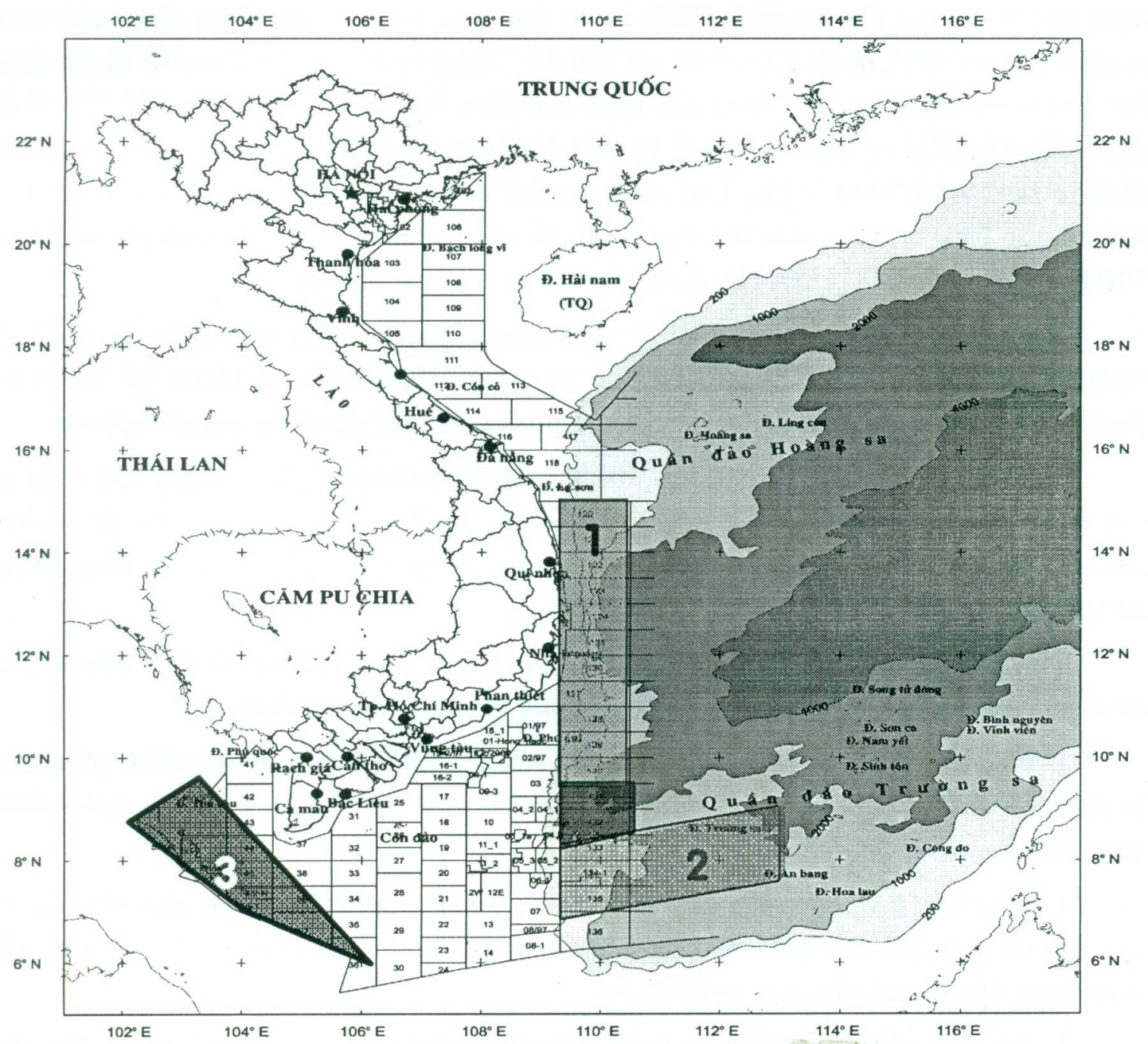

Hình 2: Vị trí bể Phú Khánh (khu vực 1), bể Tư Chính - Vũng Mây \& Tây Nam quần đảo Trường $\mathrm{Sa}$ (khu vực 2$)$, và vùng chồng lấn TLĐ Tây Nam (khu vực 3 )

Các số liệu về điều kiện tự nhiên ở độ sâu nước tới 200m được thu thập từ các kết quả $\mathrm{NCKH}$ sẵn có $[8,9]$ để ứng dụng các kết quả nghiên cứu vào điều kiện biển sâu Việt Nam.

\section{BÀI TOÁN ĐộNG LỤ̉C HỌC NGẪU NHIÊN CỦA KẾT CẤU JACKET}

\section{Phương pháp phổ giải bài toán dao động ngẫu nhiên của kết cấu Jacket $[5,11]$}

Phương trình tổng quát của bài toán động lực học một bậc tự do có dạng: 


$$
M \ddot{u}+C \dot{u}+K u=F_{(t)}
$$

Phương pháp phổ cho nghiệm của bài toán (2) dưới dạng [11]:

$$
S_{u u}(\omega)=|H(i \omega)|^{2} S_{F F}(\omega)
$$

Công thức (3) cho quan hệ: Mật độ phổ của đầu ra (phản ứng của hệ) bằng mật độ phổ của đầu vào (tải trọng) nhân với bình phương của mô đun hàm truyền, như biểu diễn trên hình 3.

Phương sai của phản ứng xác định được:

$$
\sigma_{\mathrm{u}}^{2}=\mathrm{R}_{\mathrm{uu}}(0)=\int_{0}^{\infty} \mathrm{S}_{\mathrm{uu}}(\omega) \mathrm{d} \omega=\int_{0}^{\infty}|\mathrm{H}(\mathrm{i} \omega)|^{2} \mathrm{~S}_{\mathrm{FF}}(\omega) \mathrm{d} \omega
$$
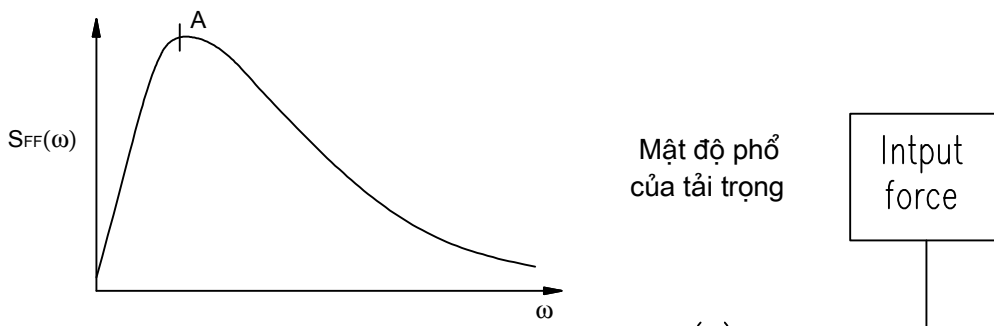

(a)

$(x)$

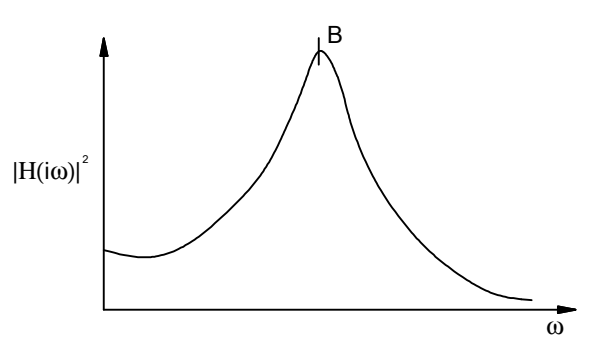

$(x)$

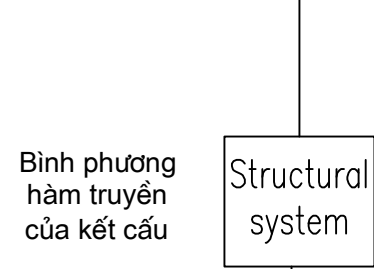

(b)
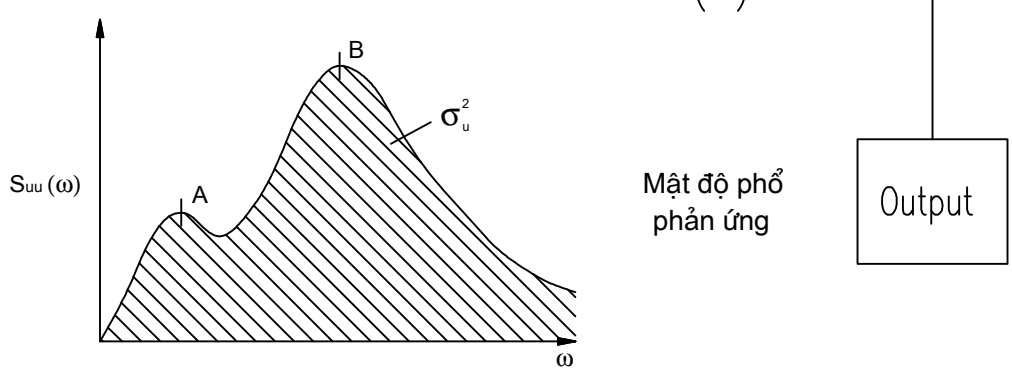

(c)

Hình 3: Mối quan hệ giữa phổ tải trọng và phổ phản ứng theo công thức (3)

Mô đun của hàm truyền $\mathrm{H}(\mathrm{i} \omega)$ có dạng : 
$|\mathrm{H}(\mathrm{i} \omega)|=\frac{1}{\mathrm{~K}} \frac{1}{\left[\left(1-\Omega^{2}\right)^{2}+(2 \xi \Omega)^{2}\right]^{1 / 2}}$

Trong đó: $\quad+\Omega=\frac{\omega}{\omega_{1}} ; \omega_{1}=(K / M)^{1 / 2}$ - tần số dao động của hệ;

$+\mathrm{C}=2 \varepsilon \mathrm{M} ; \xi=\frac{\mathrm{C}}{2 \sqrt{\mathrm{KM}}}-$ tỷ số cản .

Nếu sử dụng quan hệ giữa phổ phản ứng $\mathrm{S}_{\mathrm{uu}}(\omega)$ và phổ sóng $\mathrm{S}_{\eta \eta}(\omega)$ bởi hàm truyền dạng RAO (toán tử biên độ phản ứng, Response-Amplitude Operator), ta có dạng tương tự (3):

$\mathrm{S}_{\mathrm{uu}}(\omega)=[\mathrm{RAO}]^{2} \mathrm{~S}_{\eta \eta}(\omega)$

Bài toán động ngẫu nhiên $\mathrm{n}$ bậc tự do của kết cấu Jacket dưới dạng ma trận:

$M \ddot{U}+C \dot{U}+K U=F(t)$

trong đó:

+ M - Ma trận khối lượng của kết cấu, có kể tới khối lượng nước kèm;

+ C - Ma trận các hệ số cản do nội ma sát, có kể sức cản thuỷ động của môi trường nước;

+ K - Ma trận độ cứng của kết cấu;

+ F (t) - Vectơ tải trọng sóng ngẫu nhiên dừng, tính theo phương trình Morison dạng tuyến tính hoá và coi kết cấu là tuyệt đối cứng;

+ U, Uं và Ü - Các vectơ chuyển vị, vận tốc và gia tốc tại các nút của kết cấu.

Sử dụng phương pháp chồng các dạng dao động riêng (mode), để chuyển bài toán (7) về $n$ bài toán dạng một bậc tự do, sau đó sử dụng hàm truyền $\mathrm{H}(\mathrm{i} \omega)$ hoặc toán tử RAO, ta có phổ của phản ứng kết cấu Jacket (chuyển vị nút) phụ thuộc vào phổ của tải trọng sóng có dạng như sau [11]:

$$
S_{u_{j} u_{j}}(\omega)=\sum_{r=1}^{n} \frac{1}{M_{r}^{2}} \frac{\phi^{2}{ }_{r r}(\omega)}{\left(\omega_{r}^{2}-\omega^{2}\right)^{2}+(2 \varepsilon \omega)^{2}} S_{F_{r}^{\prime} F_{r}}(\omega) ; j=\overline{1, n}
$$

Trong đó: $S_{F_{r}^{\prime} F_{r}^{\prime}}(\omega)$ - mật độ phổ của tải trọng suy rộng xác định được theo mật độ phổ sóng :

$$
S_{F_{s} F_{s}}(\omega)=\alpha_{s} S_{\eta \eta}(\omega)
$$


Ở đây $\alpha_{\mathrm{s}}$ - hệ số phụ thuộc các yếu tố chuyển động của sóng tại vị trí tác động lên kết cấu Jacket.

Phân tích biểu thức hàm phổ chuyển vị $\mathrm{u}_{\mathrm{j}}$ của kết cấu ta thấy các số hạng trong tổng của (8) sẽ có giá trị tăng nhanh tại các vị trí của tần số $\omega=\omega_{1} ; \omega=\omega_{2} ; \omega=\omega_{3}$; và sẽ tắt dần ở một số số hạng đầu của tổng $\mathrm{n}$ số hạng. Do vậy năng lượng phổ của phản ứng cũng tập trung ở vùng lân cận tần số dao động riêng, kể từ tần số cực tiểu trở đi.

Từ (8) và (9) ta có thể thiết lập được quan hệ giữa phổ chuyển vị với phổ sóng bởi toán tử RAO dưới dạng:

$$
\mathbf{S}_{\mathbf{U j U} \mathrm{jj}}(\omega)=\left[\mathrm{RAO}_{u_{\mathrm{i}}}^{2} \mathrm{~S}_{\eta \mathrm{n}}(\omega)\right.
$$

\section{Phương pháp giải trong miền thời gian bài toán dao động ngẫu nhiên của kết cấu Jacket}

Phương pháp giải trong miền thời gian được thực hiện bằng thuật toán "rời rạc hoá" miền tần số, cho phép chuyển gần đúng từ bài toán động với sóng ngẫu nhiên có phổ $S_{\eta \eta}(\omega)$, sang một tập hợp các bài toán động tiền định với sóng Airy [15]:

$$
\eta(t)=\sum_{i=1}^{N} a_{i} \cos \left(\omega_{i}+\varphi_{i}\right)
$$

Trong đó, bộ số liệu $\left(\mathrm{a}_{\mathrm{i}}, \omega_{\mathrm{i}}, \varphi_{\mathrm{i}}\right)=$ (biên độ, tần số vòng và góc pha của sóng Airy thứ i), phụ thuộc vào các giá trị của phổ sóng $S_{\eta \eta}\left(\omega_{\mathrm{i}}\right)$, được xác định bởi kỹ thuật mô phỏng Monte Carlo (hình 4).

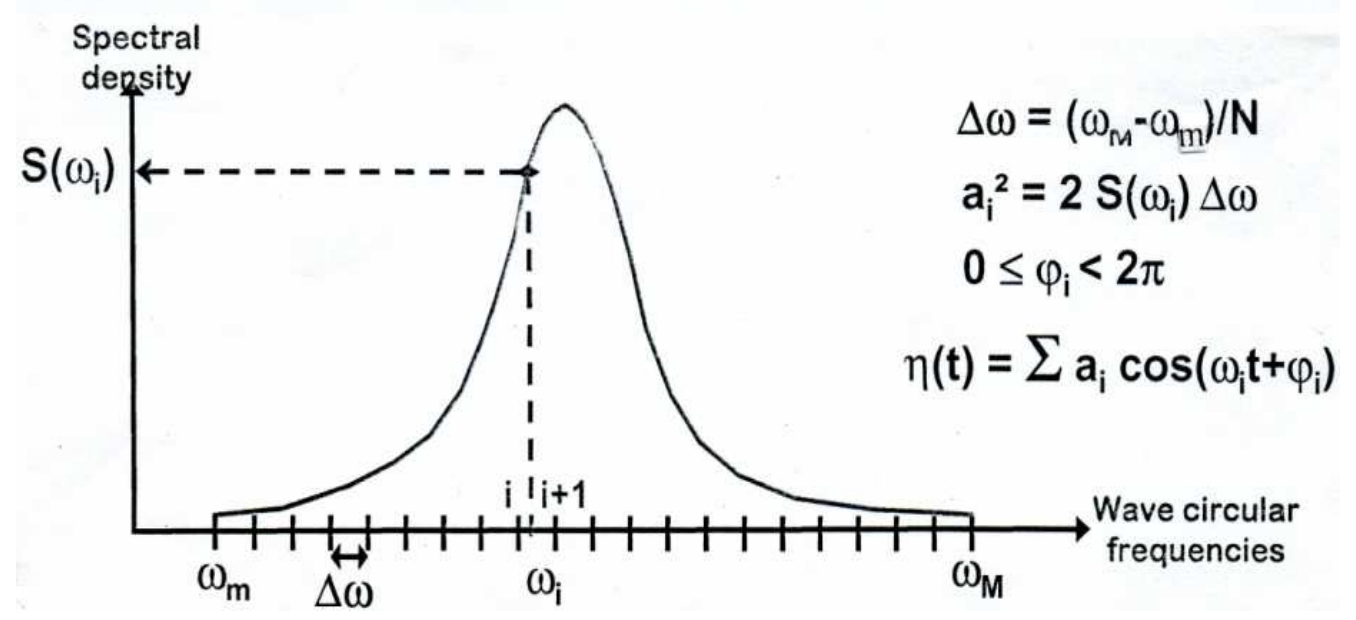

Hình 4: Rời rạc hoá miền tần số $\omega$ của phổ sóng làm nhiều đoạn [15] 
Phương pháp này khá đơn giản về thuật toán, nhưng đòi hỏi nhiều thời gian tính trên máy. Tuỳ theo yêu cầu chính xác của kết quả, người ta có thể chia miền tần số $\omega$ thành số đoạn lấy trong khoảng $\mathrm{N}=100 \div 5000$ [15].

\section{Xác định các đặc trưng xác suất của phản úng kết cấu}

Để thực hiện bài toán kiểm tra bền kết cấu Jacket theo lý thuyết độ tin cậy, cần xác định các đặc trưng xác suất của các phản ứng kết cấu là chuyển vị và ứng suất tại các vị trí cần khảo sát.

Từ hàm mật độ phổ của chuyển vị nút kết cấu, có thể xác định được các đặc tính của phổ (như độ rộng phổ) và các đặc trưng xác suất của chuyển vị, điển hình là phương sai và độ lệch chuẩn của chuyển vị.

Bình phương độ lệch chuẩn của chuyển vị (tức là phương sai) được xác định dựa trên hàm mật độ phổ (8) như sau:

$$
\sigma_{u_{j}}^{2}=\int_{0}^{\infty} S_{u_{j} u_{j}}(\omega) d \omega
$$

Hàm mật độ phổ của nội lực và ứng suất tại các phần tử kết cấu được xác định thông qua mối quan hệ đại số giữa chuyển vị nút và nội lực - ứng suất ở các vị trí cần khảo sát, từ đó xác lập được quan hệ dưới dạng hàm truyền (hoặc toán tử RAO), có dạng điển hình:

$$
\mathbf{S}_{\sigma \sigma}(\omega)=[\mathrm{RAO}]_{\sigma}^{2} \mathrm{~S}_{m}(\omega)
$$

Trong đó:

+ $\mathrm{S}_{\sigma \sigma}(\omega)$ - Hàm mật độ phổ ứng suất tại vị trí cần tính;

$+\mathrm{S}_{\eta \eta}(\omega)$ - Hàm mật độ phổ của sóng trong trạng thái biển ngắn hạn ngắn hạn cực đại thiết kế;

$+[\mathrm{RAO}]_{\sigma}=$ hàm truyền ứng suất tại điểm xét, được xác định theo phương pháp giải bài toán động lực học ngẫu nhiên trong miền tần số.

\section{KIỂM TRA BỀN KẾT CẤU JACKET THEO MÔ HÌNH XÁC SUẤT}

\section{1. Đánh giá an toàn của kết cấu Jacket theo độ tin cậy của kết cấu Jacket dựa trên điều kiện bền truyền thống}

Bài toán kiểm tra an toàn của kết cấu CTB kiểu Jacket được thực hiện theo "điều kiện bền truyền thống" xét tại các vị trí nguy hiểm của phần tử thanh ống và nút (như quy 
định trong các tiêu chuẩn thiết kế $[16,18]$ ), trong đó nội lực và ứng suất gồm 2 phần, được xác định từ các tải trọng tĩnh hoặc tựa tĩnh và từ tải trọng động của sóng trong điều kiện cực trị của môi trường biển (ULS). Tuy nhiên trong các tiêu chuẩn hiện hành nói trên, chủ yếu sử dụng mô hình tiền định đối với tác động của sóng.

Nếu chuyển động của sóng được mô tả theo các quá trình ngẫu nhiên dừng, phản ứng và nội lực động ngẫu nhiên của kết cấu Jacket được xác định như đã nêu ở mục 2. Trong trường hợp này, an toàn của kết cấu Jacket tại những vị trí đặc trưng có thể được đánh giá theo độ tin cậy của kết cấu dựa trên điều kiện bền truyền thống, có dạng [13] :

$$
\mathrm{P}=\operatorname{Prob}(\mathrm{R} \geq \mathrm{S})=\operatorname{Prob}(\mathrm{Z}=\mathrm{R}-\mathrm{S} \geq 0) \geq[\mathrm{P}]
$$

Trong đó:

$+\mathrm{R}=$ cường độ của vật liệu, có hàm mật độ xác xuất $(\mathbf{P D F})$ là $\mathrm{f}_{\mathrm{R}}$;

$+\mathrm{S}=$ ứng suất cực đại tại điểm khảo sát, có mật độ xác suất $\mathrm{f}_{\mathrm{S}}$;

+ $\mathrm{P}=$ độ tin cậy theo điều kiện bền của điểm cần kiểm tra;

+ $[\mathrm{P}]=$ độ tin cậy cho phép, hoặc có thể chấp nhận.

Tương tự (14), an toàn của kết cấu có thể được đánh giá theo điều kiện của xác suất phá huỷ:

$$
\mathrm{P}_{\mathrm{f}}=1-\mathrm{P}=\operatorname{Prob}(\mathrm{Z}=\mathrm{R}-\mathrm{S}<0)<\left[\mathrm{P}_{\mathrm{f}}\right]
$$

Trong đó: $\mathrm{P}_{\mathrm{f}}=$ xác suất phá huỷ theo điều kiện bền tại điểm xét; $\left[\mathrm{P}_{\mathrm{f}}\right]=$ xác suất phá huỷ cho phép, hay có thể chấp nhận.

Từ (14) và (15) ta thấy $\mathrm{Z}=\mathrm{R}-\mathrm{S}$ là miền an toàn theo điều kiện bền truyền thống, cũng là đại lượng ngẫu nhiên, có hàm mật độ xác suất f $\mathrm{Z}$.

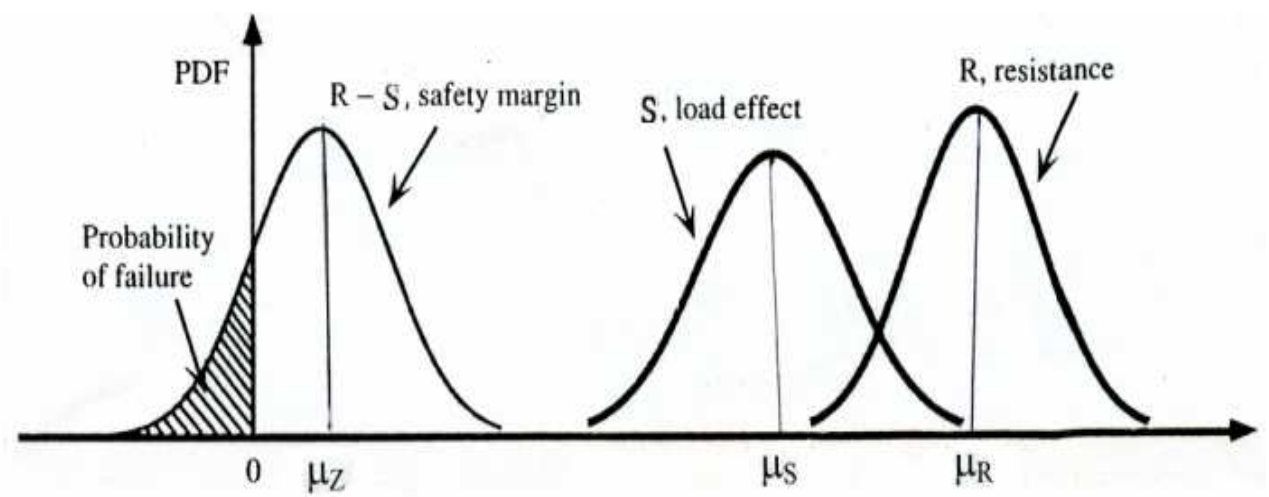

Hình 5: Đồ thị hàm mật độ xác suất của các ĐLNN $R, S$ và $Z=R-S$ 
Trên hình 5 biểu diễn đồ thị hàm mật độ xác suất ( $\mathrm{f}=\mathrm{PDF})$ của các đại lượng ngẫu nhiên (ĐLNN) $R, S$ và $Z=R-S$. Xác suất phá huỷ được thể hiện bởi diện tích miền có gạch chéo của đồ thị $\mathrm{f}_{\mathrm{R}-\mathrm{S}}=\mathrm{f}_{\mathrm{Z}}$.

Độ tin cậy còn được biểu diễn dưới dạng chỉ số độ tin cậy:

$$
\beta=\frac{\mu_{\mathrm{Z}}}{\sigma_{\mathrm{Z}}}=\frac{\mu_{\mathrm{R}}-\mu_{\mathrm{s}}}{\sqrt{\sigma_{\mathrm{R}}^{2}-\sigma_{\mathrm{S}}^{2}}},
$$

Điều kiện an toàn theo chỉ số độ tin cậy có dạng:

$\beta \geq[\beta]$,

Trong đó:

$+\mu_{R}, \mu_{S}$ và $\mu_{Z}-$ kỳ vọng toán của các $Đ L N N ~ R, S$ và $Z$;

+ $\sigma_{R}, \sigma_{S}$ và $\sigma_{Z}$ - độ lệch chuẩn của các ĐLNN $R, S$ và $Z$;

+ [ß] là chỉ số độ tin cậy cho phép, hoặc chấp nhận được.

S là ứng suất cực đại tại điểm cần kiểm tra bền, do tổ hợp các tải trọng của TTGH cực đại, trong đó chỉ có tải trọng sóng được xem là yếu tố ngẫu nhiên, nên $\mathrm{S}$ có dạng:

$$
\mathrm{S}=\mathrm{S}_{1}+\mathrm{S}_{2}
$$

Trong đó:

$+\mathrm{S}_{1}=$ đại lượng tiền định, là ứng suất tại điểm khảo sát do các tải trọng tiền định gây ra;

$+\mathrm{S}_{2}=$ đại lượng ngẫu nhiên, các ứng suất cực đại $\sigma_{\max }$ của $\sigma(\mathrm{t})$;

$+\sigma(\mathrm{t})=$ quá trình ngẫu nhiên do tải trọng sóng gây ra, có phổ ứng suất $S_{\sigma \sigma}(\omega)$, có dạng (13).

Từ hàm phổ ứng suất (13), ta xác định được luật phân phối các ứng suất cực đại $S_{2}=\sigma_{\max }$, phụ thuộc vào thông số độ rộng của phổ (là dải hẹp, rộng, hoặc bất kỳ). Biết luật phân phối của ĐLNN $S_{2}$, sử dụng các hệ thức (14) và (18), ta xác định được độ tin cậy theo điều kiện bền tại điểm xét.

Điều kiện an toàn theo độ tin cậy dựa trên điều kiện bền truyền thống (14) được biểu diễn dưới dạng tổng quát:

$$
\mathrm{P}=\operatorname{Prob}\left(\mathrm{Z}=\mathrm{g}\left(\mathrm{X}_{1}, \mathrm{X}_{2}, \ldots . \mathrm{X}_{\mathrm{n}}\right) \geq 0\right) \geq[\mathrm{P}]
$$

Trong đó: $\mathrm{Z}=\mathrm{g}\left(\mathrm{X}_{1}, \mathrm{X}_{2}, \ldots . \mathrm{X}_{\mathrm{n}}\right)=$ hàm của các $Đ L N N$, biểu diễn miền an toàn của kết cấu theo yêu cầu thiết kế, điển hình là các trạng thái giới hạn (TTGH). 
2. Đánh giá an toàn của kết cấu Jacket theo ứng suất cực đại của mô hình xác suất dựa trên điều kiện bền truyền thống

Các tiêu chuẩn hiện hành về thiết kế kết cấu CTB cố định chưa sử dụng độ tin cậy để kiểm tra bền. Tuy nhiên gần đây, một số tiêu chuẩn đã sử dung mô hình xác suất tính toán kết cấu Jacket, trong đó tác động của sóng là quá trình ngẫu nhiên dừng $[17,19]$. thực hiện kiểm tra an toàn kết cấu theo điều kiện bền truyền thống với mô hình tiền định, bằng cách tính gần đúng giá trị của ứng suất cực đại của ĐLNN max $\sigma(\mathrm{t})$ dựa trên hàm mật độ phổ của quá trình ngẫu nhiên ứng suất $\sigma(\mathrm{t})$.

Ví dụ nếu hàm phổ ứng suất thuộc loại phổ dải hẹp, tức là ĐLNN có phân phối Rayleigh, thì có thể tính được giá trị lớn nhất xấp xỉ của ứng suất trong $\mathrm{N}$ chu trình ứng suất tính với sóng của TTB cực đại (ULS) kéo dài trong thời gian $\mathrm{T}^{*}$, theo công thức [11]:

$$
\sigma_{\max }=\sqrt{\mathrm{M}_{\mathrm{o}}} \cdot \sqrt{2 \ln (\mathrm{N})}
$$

Trong đó:

$$
M_{o}=\int_{0}^{\infty} S_{\sigma \sigma}(\omega) \cdot d \omega ; N=\frac{T^{*}}{T_{Z}}=\frac{T^{*}}{2 \pi} \sqrt{\frac{M_{2}}{M_{o}}} ;
$$

với: $+\mathbf{S}_{\sigma \sigma}(\omega)=$ hàm mật độ phổ của quá trình ngẫu nhiên ứng suất $\sigma(\mathbf{t})$;

$+\mathrm{T}^{+}=$Thời gian của TTB ngắn hạn khảo sát (theo ULS) ;

$+\mathrm{T}_{\mathrm{Z}}=2 \pi \cdot \sqrt{\frac{\mathrm{M}_{\mathrm{o}}}{\mathrm{M}_{2}}}(\mathrm{sec})$.

Ví dụ với cơn bão trong 3 giờ, lấy $\mathrm{T}_{\mathrm{Z}} \approx 10 \mathrm{sec}$, ta có $\mathrm{N}=1080$ chu trình ứng suất.

\section{PHƯƠNG PHÁP LUẬN XÂY DỰNG ĐIỀU KIỆN BỀN MỞ RộNG ĐỂ XÁC ĐỊNH ĐỘ TIN CẬY TỔNG THỂ ĐÁNH GIÁ AN TOÀN CỦA KẾT CẤU JACKET}

\section{Cơ sở để xây dựng điều kiện bền mở rộng}

Theo quy định trong các tiêu chuẩn hiện hành để thiết kế các kết cấu CTB cố định $[16,17,18,19]$, việc đánh giá an toàn kết cấu Jacket (tức là kết cấu không bị phá huỷ) được thực hiện kiểm tra theo 2 điều kiện:

Điều kiện 1: là điều kiện bền, được gọi là "điều kiện bền truyền thống" (để phân biệt với “điều kiện bền mở rông” là vấn đề chính sẽ đề cập dưới đây), được thực hiện trong 1 
trạng thái biển ngắn hạn cực đại của môi trường biển với thời gian kéo dài khoảng 3h hoặc 6h (thường sử dụng với tần suất 100 năm, như quy định trong các Tiêu chuẩn hiện hành), còn gọi là phép kiểm tra trong TTGH cực đai (ULS); Việc kiểm tra kết cấu theo điều kiện 1 không phụ thuộc vào thời gian khai thác $\mathrm{CTB}$, được thực hiện trong trạng thái giới hạn (TTGH) cực đại (ULS);

Điều kiện 2: là điều kiện mỏi, được thực hiện để đánh giá tổn thất của kết cấu do phá huỷ mỏi tích luỹ trong quá trình khai thác $\mathrm{CTB}$; Việc kiểm tra kết cấu theo điều kiện 2 hoàn toàn phụ thuộc vào thời điểm khai thác $\mathrm{CTB}$, được thực hiện trang TTGH mỏi (FLS); có nghĩa là thời gian khai thác $\mathrm{CTB}$ càng dài, thì tổn thất kết cấu càng lớn; cho đến lúc tổn thất gây phá huỷ kết cấu, đó chính là mốc thời gian để xác định "tuổi thọ mỏi” của kết cấu CTB.

Nhận xét: Việc kiểm tra bền truyền thống (điều kiện 1) được thực hiện độc lập với kiểm tra mỏi (điều kiện 2) để đánh giá an toàn của kết cấu CTB. Khiếm khuyết đáng kế ở đây là cách tính theo các tiêu chuẩn thiết kế đã không kế đến sự tương quan chặt chẽ giữa 2 điều kiện phá huỷ do bền và phá huỷ do mỏi. Cụ thể là, khi tác động của môi trường cực trị lên kết cấu ở thời điểm $\mathrm{t}$, thì hiện trạng kết cấu không còn nguyên vẹn nữa, mà kết cấu đã bị tổn thất do phá huỷ mỏi tích lũy trong khoảng thời gian là từ khi bắt đầu khai thác đến thời điểm $\mathrm{t}$. Khiếm khuyết này có thể giải thích là với mô hình tiền định không thể đánh giá định lượng được tổn thất mỏi ảnh hưởng đến điều kiện bền của kết cấu.

Sau đây sẽ trình bày cách khắc phục khiếm khuyết trên nhờ lý thuyết độ tin cậy cho phép đánh giá tương tác giữa 2 trạng thái phá huỷ kết cấu.

2. Xây dựng điều kiện bền mở rộng để xác định độ tin cậy tổng thể đánh giá an toàn của kết cấu Jacket

\section{1. Điều kiện bền mở rộng}

1) Sư kiện an toàn về bền khi chịu bão thiết kế (sự kiện $\mathrm{A}$ ): là sự kiện được xác định bới TTGH cực đại, có ký hiệu là "ULS", được gọi là mặt giới hạn thứ 1 theo nghĩa của lý thuyết độ tin cậy [13];

2) Sụ kiện an toàn về phá huỷ mỏi tích luy (sự kiện B): là sự kiện được xác định bởi TTGH mỏi, có ký hiệu là "FLS", được gọi là mặt giới hạn thứ 2 theo nghĩa của lý thuyết độ tin cậy [13];

3) Điều kiện bền mở rộng đưọc xây dụng tù̀ sụr kiện an toàn về bền luôn cùng xuất hiện với sụr kiện an toàn về mỏi (sự kiện $\mathrm{C}$ ): được thể hiện bởi các tính chất sau:

+ Tính chất 1: Trong quá trình khai thác CTB, bất cứ lúc nào có bão xẩy ra (sự kiện 
$\mathrm{A})$, thì kết cấu đã chịu tổn thất mỏi (sự kiện $\mathrm{B})$, tức là $\mathrm{A}$ và $\mathrm{B}$ không xung khắc và tồn tại sự kiện $\mathrm{C}$ : $\mathrm{C}=\mathrm{AB}$;

+ Tính chất 2: Hai sự kiện $\mathrm{A}$ và $\mathrm{B}$ là độc lập (không tương quan): thực tế thống kê cho thấy 2 sự kiện này không có ràng buộc gì với nhau.

Sự kiện $\mathrm{C}$ được xây dựng như trên được gọi là sự kiện an toàn của kết cấu theo “điều kiện bền mở rộng".

\section{2. Độ tin cậy tổng thể của kết cấu Jacket}

Từ tính chất 1 cho xác suất của sự kiện $\mathrm{C}$ được xác định bằng xác suất của giao giữa 2 sự kiện $A$ và $B$ :

$\mathrm{P}(\mathrm{C})=\mathrm{P}(\mathrm{A} \cap \mathrm{B})=\mathrm{P}(\mathrm{AB}) \neq 0$;

Từ tính chất 2 ta có thể viết :

$\mathrm{P}(\mathrm{A} / \mathrm{B})=\mathrm{P}(\mathrm{A})$ và $\mathrm{P}(\mathrm{B} / \mathrm{A})=\mathrm{P}(\mathrm{B})$

Theo định lý nhân xác suất, kể đến (21) và (22), ta có:

$\mathrm{P}(\mathrm{C})=\mathrm{P}(\mathrm{A} \cap \mathrm{B})=\mathrm{P}(\mathrm{A} / \mathrm{B}) \cdot \mathrm{P}(\mathrm{B})=\mathrm{P}(\mathrm{A}) \cdot \mathrm{P}(\mathrm{B} / \mathrm{A})$

Suy ra: $\mathrm{P}(\mathrm{C})=\mathrm{P}(\mathrm{A}) . \mathrm{P}(\mathrm{B})$,

Trong đó:

$+\mathrm{P}(\mathrm{C})$ là độ tin cậy tổng thể của kết cấu Jacket (tại điểm khảo sát), khi xét đồng thời về điều kiện an toàn về bền và phá huỷ mỏi, có ký hiệu là $\mathrm{P}_{\mathrm{T}}$;

$+\mathrm{P}(\mathrm{A})$ ký hiệu là $\mathrm{P}_{\mathrm{OB}}=$ const là độ tin cậy dựa trên điều kiện bền truyền thống (14);

$+\mathrm{P}(\mathrm{B})$ ký hiệu là $\mathrm{P}_{\mathrm{m}}(\mathrm{t})=$ là độ tin cậy do tổn thất mỏi tích luỹ trong quá trình khai thác CTB [2].

Thay các ký hiệu trên vào (23) ta có biểu thức xác định độ tin cậy tổng thể của kết cấu tại điểm khảo sát là tích của độ tin cậy bền và độ tin cậy phá huỷ mỏi:

$$
\mathrm{P}_{\mathrm{T}}(\mathrm{t})=\mathrm{P}_{\mathrm{oB}} \cdot \mathrm{P}_{\mathrm{m}}(\mathrm{t})
$$

Từ kết quả (24) cho thấy độ tin cậy tổng thể của kết cấu Jacket (tại vị trí khảo sát) dựa trên «điều kiện bền mở rộng », là hàm đơn điệu giảm theo thời gian, phản ảnh đúng hiện trạng về khả năng chịu lực của kết cấu bị suy giảm theo thời gian do tổn thất phá huỷ mỏi tích luỹ.

Trên hình 6 biểu diễn đường độ tin cậy về bền $\mathrm{P}_{\mathrm{OB}}=$ const, đường cong độ tin cậy tổng cộng $\mathrm{P}_{\mathrm{T}}(\mathrm{t})$, đường cong xác suất phá huỷ tổng cộng $\mathrm{P}_{\mathrm{f}} \mathrm{T}(\mathrm{t})=1-\mathrm{P}_{\mathrm{T}}(\mathrm{t})$, và đường cong 
phá huỷ mỏi $\mathrm{P}_{\mathrm{fm}}(\mathrm{t})$.

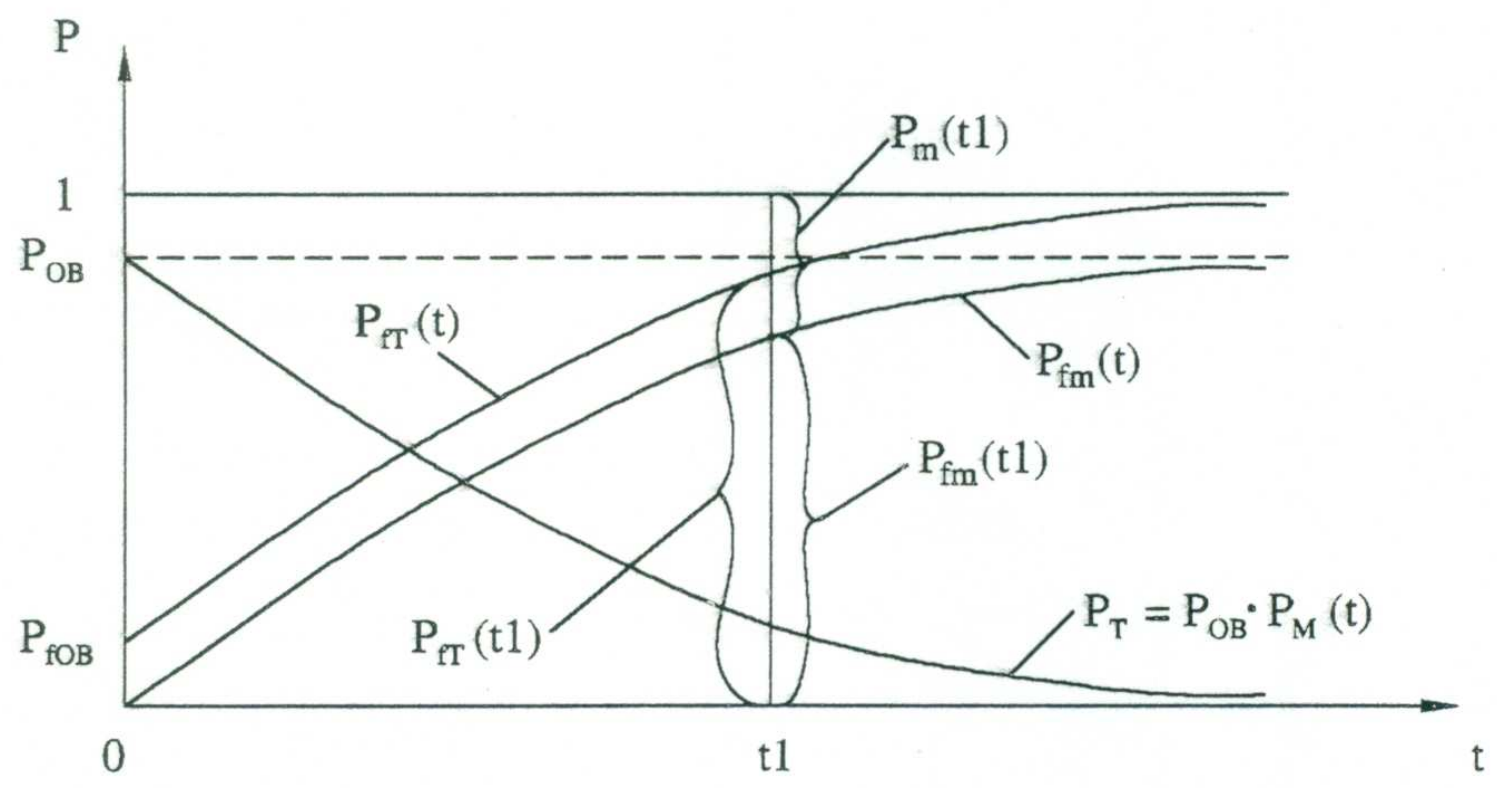

Hình 6: Các đường cong biểu diễn độ tin cậy tổng cộng và xác suất phá huỷ tổng cộng

\section{V. ÁP DỤNG CHO ĐIỀU KIỆN BIỂN VIỆT NAM}

\section{Lựa chọn cấu hình kết cấu Jacket ở độ sâu nước $200 \mathrm{~m}$, Bắc bể Nam Côn Sơn}

Sử dụng các số liệu về điều kiện tự nhiên trong các tài liệu [6, 9] và lựa chọn cấu hình từ tài liệu [3], ta sẽ thực hiện tính toán cho kết cấu Jacket có sơ đồ như trên hình 7 dưới đây. 

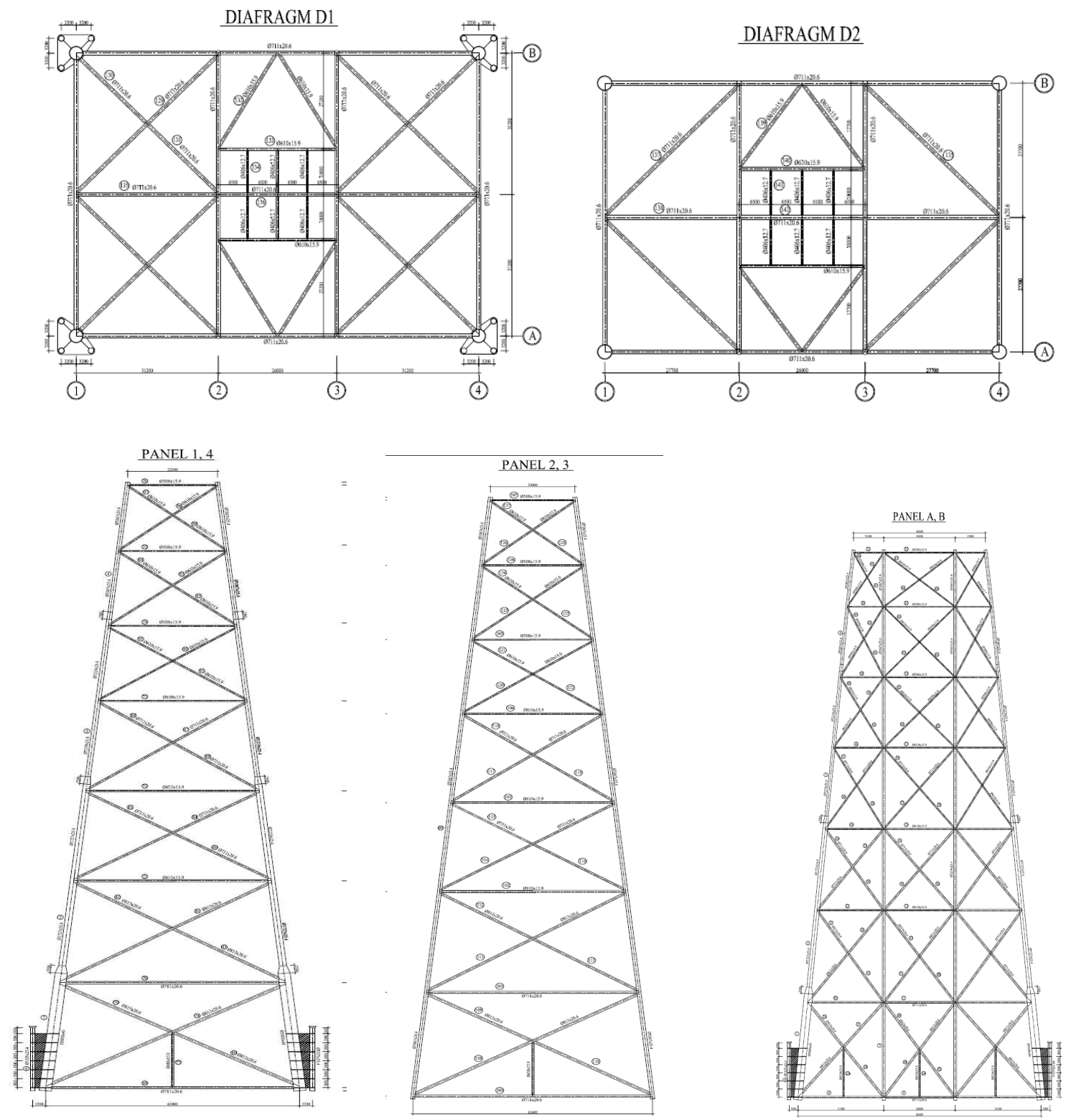

Hình 7: Cấu hình của kết cấu Jacket ở độ sâu nước 200 m

\section{Kết quả chính của bài toán bền và mỏi}

Thực hiện tính theo Tiêu chuẩn API [16] và sử dụng phần mềm chuyên dụng SACS. Dưới đây là trích dẫn một số kết quả tiêu biểu. 
1) Kết quả kiểm tra bền thanh điển hình

\begin{tabular}{|c|c|c|c|}
\hline Tên thanh & Vị trí & $\begin{array}{c}\text { Hệ số sử dụng } \\
\text { theo mô hình } \\
\text { động tiền định }\end{array}$ & $\begin{array}{c}\text { Hệ số sử dụng theo } \\
\text { mô hình động ngẫu } \\
\text { nhiên }\end{array}$ \\
\hline $469-293$ & $\begin{array}{c}\text { Thanh nhánh khoang } \\
\text { cuối }\end{array}$ & 0.96 & 1.02 \\
\hline $195-295$ & Cọc phụ nối đất & 1.05 & 1.07 \\
\hline
\end{tabular}

2) Kết quả kiểm tra bền nút điển hình

\begin{tabular}{|c|c|c|c|}
\hline Tên nút & Vị trí & $\begin{array}{c}\text { Hệ số sử dụng theo mồ } \\
\text { hình động tiền định }\end{array}$ & $\begin{array}{c}\text { Hệ số sử dụng theo mô } \\
\text { hình động ngẫu nhiên }\end{array}$ \\
\hline 62 & $\begin{array}{c}\text { Nút khoang 2, } \\
\text { mặt } \mathrm{x}=-13 \mathrm{~m}\end{array}$ & 0.92 & 0.95 \\
\hline 63 & $\begin{array}{c}\text { Nút khoang 2, } \\
\text { mặt } \mathrm{x}=13 \mathrm{~m}\end{array}$ & 0.93 & 0.97 \\
\hline
\end{tabular}

3) Bài toán mỏi tiền định

\begin{tabular}{|c|c|c|c|}
\hline Nút & Thanh & Tổn thất tích lũy 1 năm & Tuổi thọ mỏi \\
\hline 907 & $907-1007$ & 0.0296 & 33.8 \\
\hline 913 & $913-1013$ & 0.0492 & 20.3 \\
\hline
\end{tabular}

4) Bài toán mỏi ngẫu nhiên

\begin{tabular}{|l|c|c|c|}
\hline Nút & Thanh & Tổn thất tích lũy 1 năm & Tuổi thọ mỏi \\
\hline 907 & $907-1007$ & 0.0326 & 30.7 \\
\hline 913 & $913-1013$ & 0.0541 & 18.5 \\
\hline
\end{tabular}

3. Nhận xét: Tính theo mô hình tiền định và mô hình xác suất cho kết quả khá gần nhau.

\section{KẾT LUẬN}

Bài báo đã trình bày cơ sở phương pháp luận đánh giá an toàn của kết cấu CTB cố định kiểu Jacket theo mô hình xác suất và lý thuyết độ tin cậy dựa trên điều kiện bền mở 
rộng, tức là điều kiện bền có kể đến sự suy giảm khả năng chịu lực của kết cấu do tổn thất mỏi tích luỹ trong quá trình khai thác công trình. Trong phần ứng dụng vào điều kiện thực tế của biển sâu Việt Nam, đã thực hiện tính toán theo mô hình xác suất dựa theo tiêu chuẩn API và $\mathrm{DNV}[17,19]$.

Dựa trên phương pháp luận đã được xây dựng, có thể tiếp tục thực hiện các tính toán theo điều kiện bền mở rộng để minh hoạ việc đánh giá an toàn theo độ tin cậy tổng thể cho kết quả sát với thực tế, do vậy sẽ đảm bảo thiết kế kết cấu CTB được an toàn hơn so với cách tính truyền thống theo các tiêu chuẩn thiết kế hiện hành.

Các nghiên cứu này cũng có thể áp dụng mở rộng để đánh giá an toàn cho các loại CTB nói chung, như trong nghiên cứu trình tại bài báo [4] do tác giả đã báo cáo ở Hội nghị Quốc tế Kobe (Nhật Bản).

Mặt khác, có thể đề nghị xem xét đưa các kết quả nghiên cứu này bổ sung vào tiêu chuẩn thiết kế cho các loại công trình biển để nâng cao an toàn cho công trình, nhất là trong bối cảnh biến đổi khí hậu bất thường toàn cầu.

\section{TÀI LIỆU THAM KHẢO}

1. Phạm Khắc Hùng, Mai Hồng Quân, Vũ Đan Chỉnh, 2009. Đánh giá an toàn công trình biển cố định bằng thép chịu tải trọng ngẫu nhiên ở vùng nước sâu $200 \mathrm{~m}$ áp dụng vào điều kiện biển Việt Nam. Tuyển tập các công trình NCKH Chương trình Biển KC.09/06-10, Hội thảo "Địa chất và Công trình biển”, Hoà Bình, 4-6/12/2009, Tr.119- 179.

2. Pham Khac Hung, Mai Hong Quan, Ta Thi Hien (ICOFFSHORE.HUCE \& TCU), 2010. Assessment of fatigue damage of fixed offshore steel structures installed in deepwater areas of Vietnam sea by probabilistic modelling. PETROVIETNAM Journal, Vol 6 - 2010, PP.55-60.

3. Mai Hong Quan, Vu Dan Chinh (ICOFFSHORE.HUCE), 2010. The basis of structural solution selection for fixed offshore steel structures at $200 \mathrm{~m}$ water depth in Vietnam sea conditions. PETROVIETNAM Journal, Vol 6 - 2010, PP.61-68.

4. Pham Khac Hung, Dinh Quang Cuong, Mai Hong Quan, Nguyen Van Ngoc (ICOFSHORE \& VMU, Vietnam), 2004. Estimation of the Total Reliability of Offshore Structures in Viertnam sea Conditions Combining the Ultimate Limit States and Fatigue Limit States. OCEANS'04 MTS/IEE Conference Proceedings, Kobe, Japan, Nov.2004, PP.176-184. 
5. Phạm Khắc Hùng, 2008. Xây dựng phương pháp luận tính toán thiết kế công trình biển cố định bằng thép đến độ sâu $200 \mathrm{~m}$ nước để phát triển khả năng của VSP trong lĩnh này. (153 trang). Hợp đồng NCKH - Vietsovpetro. Lưu trữ tại Viện XDCT Biển ĐHXD.

6. Đinh La Thăng (Chủ tịch Tập đoàn Dầu khí QGVN), 2007. Xây dựng Tập đoàn Dầu khí QGVN trở thành Tập đoàn kinh tế mạnh của đất nước. Tạp chí Dầu khí, Số 1-2007,Tr.4-7.

7. Nguyễn Huy Quý, 2006. Nghiên cứu cấu trúc địa chất và địa động lực làm cơ sở đánh giá tiềm năng dầu khí ở các vùng biển sâu và xa bờ của Việt Nam. Báo cáo kết quả NCKH Đề tài cấp NN KC.09.06, “Tuyển tập các kết quả chủ yếu của Chương trình KC.09, Tập I".

8. Nguyễn Mạnh Hùng, Nguyến Thế Tưởng, Bùi Mạnh Hoà, 2008. Xác định bộ số liệu về điều kiện môi trường vùng bể nước sâu Nam Côn Sơn phục vụ việc lập Luận chứng KHKT-KT thiết kế CTB vùng nước sâu TLĐ.VN. ĐT. KC.09.15/06-10. Tuyển tập các công trình NCKH, Hội thảo "Địa chất và Công trình biển", Hoà Bình, 46/12/2009, Tr.289-312.

9. Phạm Văn Ty, Nguyễn Viết Tình và Phạm Thị Việt Nga (ĐH. Mỏ ĐC), 2008. Địa hình công trình vùng Đông Bắc bể Nam Côn Sơn. Đề tài cấp NN KC.09.15/06-10.

10. Lindsey Wilhoit and Chad Supan (Mustang Engineering), 2008. 2008 Deepwater Solutions \& Records for Concept Selection. Offshore Magazine, PennWell,.May 2008, Houston, USA.

11. Barltrop NDP, Adams AJ, 1991. Dynamics of Fixed Marine Structures; Ch.11Strength and Fatigue. ButterWorth Heinemann - UK.

12. OEP Monash University, 1993. Design, Construction \& Installation of Steel Jacket Structures. Intensive Short Course, Australian Maritime Eng. Coop. Research Centre.

13. Palle Thoft-Christensen, Michael J.Baker, 1982. Structural Reliability Theory and Its Applications. Springer-Verlag Berlin Heidelberg New York.

14. OEP Monash University, 1992. Safety and Reliability of Offshore Structures. Intensive Course, Australian Maritime Eng. Coop. Research Centre.

15. Bureau Veritas - MCS. Theory Manual Part1: General \& Time domain Simulation. Ariane-3Dynamic, Version 6.3. 
16. API-RP2A-WSD, 2002. Recommended Practice for Planning, Designing and Constructing Fixed Offshore Platforms - Working Stress Design. American Petroleum Institute, Washington, D.C., 21rst Ed.

17. API-RP2A-LRFD, 1997. Recommended Practice for Planning, Designing and Constructing Fixed Offshore Platforms - Load and Resistance Factor Design. American Petroleum Institute, Washington, D.C., 1rst Ed.

18. DNV, 1993. Rules for Classification of the Fixed Offshore Installation.

19. DNV, 2004. Design of offshore steel structures, General (LRFD Method).

\title{
ESTABLISHING THE EXPANDED STRENGTH CONDITION FOR DETERMINATION OF THE TOTAL RELIABILITY ASSESSING THE SAFETY OF FIXED OFFSHORE STEEL STRUCTURES, AND ITS APPLICATION IN DEEPWATER SEA CONDITION OF VIETNAM
}

\author{
PHAM KHAC HUNG
}

Summary: This paper presents a new approach to assess the safety of fixed offshore structures of Jacket type for oil and gas exploitation in deepwater areas, basing on the total structural reliability determined by "the expanded strength condition", that is the one in taking into account of the real structural state in damage due to fatigue cumulative during operating process.

With the expanded strength condition, the safety assessment of structures subjected to random wave loading by the total reliability will give the more exact result than the one with the traditional strength condition corresponding to the current standards. This assessment is particularly efficient for deepwater platform structures.

The research result was applied in first step to practical condition of $200 m$ water depth of Nam Con Son Basin, South-East Continental Shelf of Vietnam. This paper is taken from research results of the National Research Project KC.09.16/06-10 managed by the author who performed directly it with the collaboration of some ICOFFSHORE's colleagues.

Ngày nhận bài: 20 - 8 - 2010

Ngườ nhận xét: PGS. TS. Đinh Quang Cường 